\title{
Multi-access edge computing enabled internet of things: advances and novel applications
}

\author{
Rongbo $\mathrm{Zhu}^{1} \cdot \mathrm{Lu} \mathrm{Liu^{2 }} \cdot$ Houbing Song ${ }^{3} \cdot$ Maode $\mathrm{Ma}^{4}$
}

Published online: 3 August 2020

(C) Springer-Verlag London Ltd., part of Springer Nature 2020

\begin{abstract}
Multi-access edge computing (MEC) is emerging as a new and promising methodology with the development of Internet of Things (IoT) and smart devices, which enables a large class of applications and has emerged with a significance to change our life, and also is raising the challenging research issues due to the requirements of low latency, deployment independence, location awareness and mobility support. However, recent advances on distributed artificial intelligence (AI), big data, and edge/terminal cooperation schemes show that IoT still struggles with fundamental, long-standing problems, such as computational models, resource allocation and scheduling, power efficient data processing and cooperative decisionmaking mechanisms in edge. How to enhance IoT with MEC, distributed AI, cooperation and novel applications is worth exploring. The objective of this special issue is to report high-quality state-of-the-art research contributions that address these key aspects of MEC-enabled IoT and novel applications. By presenting a selection of papers on various topics related to MEC-enabled IoT, we hope to shed light on the multiple aspects of this emerging paradigm. The papers included in this issue propose solutions for resource management and allocation, multimedia processing, as well as novel applications.
\end{abstract}

Keywords Internet of things $\cdot$ Multi-access edge computing $\cdot$ Novel applications

\section{Introduction}

With the rapid growth of communication-intensive mobile applications, smart cities and Internet of Things (IoT) are raising the challenging research issues due to the requirements of low latency, deployment independence, location awareness and mobility support [1]. We need better

\footnotetext{
Rongbo Zhu

rbzhu@mail.scuec.edu.cn

Lu Liu

1.liu@leicester.ac.uk

Houbing Song

Houbing.Song@erau.edu

Maode Ma

emdma@ntu.edu.sg

1 South-Central University for Nationalities, Wuhan, China

2 University of Leicester, Leicester, UK

3 Embry-Riddle Aeronautical University, Daytona Beach, FL, USA

4 Nanyang Technological University, Singapore, Singapore
}

computing power and service capabilities to make the IoT intelligent and optimize cloud-edge-terminal ecosystem for tackling network bottleneck. Multi-access edge computing (MEC) offers application developers and content providers cloud-computing capabilities and an information technology (IT) service environment at the edge of the network $[2,3]$, and a new ecosystem and value chain are provided by MEC enabled IoT, which allows operator to flexibly and rapidly deploy innovative applications and intelligent services towards mobile subscribers, enterprises and vertical segments [4]. Despite numerous benefits from MEC, there are still some challenges in MEC-enabled IoT.

MEC-enabled IoT integrates and merges multidiscipline. Considering that MEC-enabled IoT embodies distributed cooperation and learning intelligence [5, 6], and has the characteristic of integrating past experiences into itself, it comes to an emerging paradigm of distributed intelligent computing methodologies and has been one of the most popular trends in smart industry, agriculture, healthcare, home, transportation, etc.. Since MEC-enabled IoT provides novel distributed computing and processing ability and enables rapid machine-to-machine 
communication and machine-to-human interaction, edge intelligence assisted IoT takes localized processing farther away from the network right down to the sensor by pushing the computing processes even closer to the data sources, which also provides multidisciplinary novel solutions and interactions to improve life quality and user experiences.

It is obvious that MEC-enabled IoT promotes a large class of applications and has emerged with a great potential to change our life and improve user's experience. However, recent advances on artificial intelligence (AI), cloud/fog computing, big data, and novel communication techniques show that MEC-enabled IoT still struggles with fundamental, long-standing problems. How to enhance distributed computing and processing performance with distributed machine learning (ML), common sense, intelligent interaction, data security and novel applications is worth exploring.

This special issue aims to report high-quality research on recent advances toward the realization of the MECenabled computing paradigm and MEC-enabled IoT applications. Our goal is to shed light in the multifaceted aspects of this emerging paradigm, including solutions for theoretical foundation, system model, reliability, safety, data analytics, data management, intelligent services, as well as novel applications to building smart cities and society. After a rigorous and careful review process performed by reviewers and the Guest Editors, 8 papers were accepted addressing subjects that were classified in the following categories: (1) resource management and allocation; (2) multimedia processing; and (3) MEC-enabled novel applications. The papers included in this special issue are categorized into following areas.

\section{Content}

\subsection{Resource management and allocation}

MEC complements the IoT paradigm in terms of high scalability, low delay, location awareness, and allowing of using local client computing capabilities in real time [6]. As a new paradigm to offload computation tasks from the cloud to the edge, unlike traditional cloud computing, in which tasks are offloaded to remote cloud datacenters, MEC assigns computation tasks to multiple edge nodes that are deployed close to end users [3, 6]. Thus, MEC is capable of reducing data transmission and network traffic between cloud servers and IoT devices. However, compared with the traditional centralized caching, resource management and allocation in MEC-enabled IoT are relatively complex and advanced.

Meanwhile, heterogeneous networks and edge caching are two key technologies to meet the ever-growing wireless data demand by increasing the regional spectral efficiency, decreasing the transmission delay and avoiding the use of the limited backhaul capacity. Focusing on a time and space evolution cache refreshing in multi-cluster heterogeneous networks, Yang et al. [7] present a two-step content placement probability optimization scheme. At the initial complete cache refreshing optimization, the joint optimization of the activated base station density and the content placement probability is considered. And the optimization problem is formulated into a GP problem. At the following partial cache refreshing optimization, the time-space evolution also is considered, which derives a convex optimization problem subjected to the cache capacity constraint and the backhaul limit constraint. And the redundant information in different content popularity is used based on the deep neural network to avoid the repeated calculation because of the change in content popularity distribution at different time slots. The scheme provides the great approximation to the optimum and generalization ability.

Limited computing capacity and energy is a critical issue in IoT, especially in big data and time sensitive applications. Many typical application scenarios involve the $5 \mathrm{G}$ technology, such as the applications in dense residential areas, offices, stadiums, subways, etc., and the various applications could be augmented reality, virtual reality, ultra-high definition video, cloud storage, etc., which in general require for high speed and low latency. Efficient handle offload is a challenge in MEC-enabled IoT. In order to solve offload effectively, Tang et al. [8] explore the time and energy consumption in user experience and then formulate a mixed overhead of time and energy minimization problem, which is a nonlinear programming problem. And the block coordinate descent method to deal with each variable step by step is adopted. Then the minimum value of delay parameters in the model is evaluated in two special cases: 1-offloading and 0-offloading. The necessary and sufficient conditions for the existence of two special cases are also deduced. Besides, the multi-user situation is also discussed. The proposed partial offload strategy enables the edge devices to process more traffic with less power and time.

\subsection{Multimedia processing}

The amount of data generated in IoT is increasing dramatically, which presents a higher requirement for computing and storage resources, and even intelligent processing. As a new paradigm to process multimedia data at the edge of the network, MEC-enabled IoT decentralizes tasks. In MEC paradigm, plenty of edge servers are established close to IoT devices to deal with the requests from these devices before they are routed to the core 
network $[1,5]$. Hence, the computation and transmission between devices and cloud server can be partly migrated to edge servers or cloud server, which enables IoT devices to fulfill complex data processing and analysis tasks with lower latency, higher performance, and less energy consumption by taking advantage of the services deployed on edges. And the edge servers can work co-operatively to get full control and improve the offline scheduling capability at edge side. Besides, with the help of edge servers in proximity, applications are enabled to learn from the mobile users' real-time context information to improve data processing performance and quality of experience of users.

In many scenarios, satellites play a key role in ground exploration, weather forecasting, and disaster warning, and exist as a carrier of information transmission in the era of IoT. Concentrating on massive satellite data processing in imaging mission planning and scheduling, Song et al. [9] present a general data-driven imaging satellite mission planning framework, which mainly includes three parts: task assignment, planning and scheduling and task execution. The two core parts of the planning and scheduling module are machine learning algorithms and planning and scheduling algorithms, which greatly affect the quality of the results. Machine learning algorithm is mainly used to quickly obtain feasible initial solution. This idea can be used to quickly analyze and model the imaging satellite observation mission planning, imaging satellite measurement and control, data downlink mission planning problems. It has a strong generality and is suitable for most situations of imaging satellites. The framework provides a general solution to the problem of satellite mission planning.

Robot technology has become an important symbol to measure the level of scientific technological innovation and high-end manufacturing. Visual simultaneous localization and mapping (SLAM) is the key step for mobile robots to perform tasks independently and perfectly. However, SLAM and laser-based SLAM are not widely used, limited by the computational complexity, data processing and very low and predictable latency. In order to improve the data processing performance, $\mathrm{Li}$ et al. [10] present an edge computing-based accelerate scheme, which design an inertial unit mobile robotic navigation system, and all sensors are connected in edge layers. The accelerometer data are integrated using the Kalman filter data fusion algorithm to filter the random drift error caused by the gyroscope and the electronic compass. Meanwhile a lowcost distance sensor is used to detect the depth and upload to the other fog node for computation. And the $3 \mathrm{D}$ point coordinate information is projected onto the two-dimensional coordinate extraction feature point to establish the feature map. The extended Kalman filter SLAM is used to achieve simultaneous positioning and mapping.

\subsection{MEC-enabled novel applications}

With the advances of 5G, MEC-enabled IoT will allows operator to flexibly and rapidly deploy innovative applications and various intelligent services, especially with the latest innovation in AI, blockchain and big data techniques, which further envisions the future IoT to create inherent intelligence.

The cold-chain logistics efficiency of fresh agricultural product remarkably is improved with the application of IoT. However the operating costs inevitably rise. In order to decrease operating costs in cold-chain logistics, Sun et al. [11] explore the significant boundary value related to the revenue decision of cold-chain logistics of fresh agricultural product, which analyzes the impact of the adoption of IoT on upstream and downstream wholesale prices, retail price, and order quantity decision based on the costs and revenues. And several decision-making models are proposed to analyze the performance of different factors. And the scheme provides a scientific basis for investment in the IoT by enterprises engaged in cold-chain operation of fresh agricultural products.

The widespread popularization of Internet has brought about various Internet-based business models. Similarly, the application of IoT is fermenting IoT-based business models in various fields, and IoT is reshaping the business process in various industries such as transportation, manufacturing, healthcare, and agriculture. Focusing on smart applications, Ruan et al. [12] present an IoT-based e-business model of intelligent vegetable greenhouses with details on the basic process and key nodes of the e-business model. A system framework of IoT-based intelligent vegetable greenhouses is formulated, with specific analysis on the different roles of greenhouse farmers, agricultural specialists, system administrators and consumers in the IoT system. Operations management issues in the IoT-based e-business model are recognized and corresponding mathematical models are formulated to minimize operation costs or maximize vegetable revenues, which is helpful for greenhouses to focus on main activities in the business model. Moreover, key operation issues are recognized including big-data-driven pricing, planting structure and time optimization, water and fertilizer integrated control, plant light supplement, and order-driven picking and packing. The characteristics brought about by IoT techniques to these operation issues are analyzed, which provides a novel MEC-enabled IoT application.

In existing overflow remote intelligent monitoring system, a huge amount of data uploading and multiple processing brings great challenges to the bandwidth load and real-time feedback in IoT. In order to monitor overflow in the oil and gas well drilling process, Liang et al. [13] 
propose a MEC-based overflow intelligent monitoring system, which utilizes MEC to provide real-time local services for field devices, and it can reduce the data uploading amount by preliminarily analyzing the computing tasks of the cloud computing platform. And the information entropy theory has been adopted to improve fuzzy c-means clustering algorithm to overcome the disadvantage that the user gives the number of clustering actively. Considering the correlation between the occurrence of overflow accident and the changing trend of standpipe pressure and casing pressure, an intelligent early warning model of drilling overflow accident is proposed based on information entropy, which adopts the adaptive determination of the number of clusters for clustering to improve the accuracy and reliability of the overflow warning.

With the advancement of software technique, complex IoT applications have been implemented as a set of lightweight and independent micro-services. To achieve abundant sharing, lightweight micro-services are distributed among containers over the mobile edge network. How to produce the optimal collocation of suitable micro-service for an application in MEC environment is an important issue that should be addressed. To address this issue, Zhou et al. [14] propose a latency-aware micro-service mashup approach. The problem is formulated into an integer nonlinear programming. Then, the NP-hardness of the problem is proved by reducing it into the delay constrained least cost problem. And an approximation latency-aware microservice mashup approach is presented to solve the problem. And the proposed approach can achieve a substantial reduction in network resource consumption while still ensuring the latency constraint.

\section{Conclusions}

In conclusion, the papers included in this special issue show the diversity of research being conducted in the field of MEC-enabled IoT and novel applications. While this issue offers limited contributions to this field, we strongly believe that it has opened the door for more involved research in this important area of research.

Acknowledgements The guest editors would like to thank all the authors and the reviewers for their hard work and contributions in helping to organize this special issue. They also would like to express their heartfelt gratitude to the Editor-in-Chief, Prof. John MacIntyre, for giving us this great opportunity, and the members of the Editorial Staff for their support during the process.

\section{References}

1. Guo S, Wang K, Pau G, Rayes A (2019) Edge intelligence for the industrial internet of things. IEEE Netw 33(5):4

2. Elazhary H (2019) Internet of Things (IoT), mobile cloud, cloudlet, mobile IoT, IoT cloud, fog, mobile edge, and edge emerging computing paradigms: disambiguation and research directions. J Netw Comput Appl 128:105-140

3. Zhang Y, Huang H, Yang L-X, Xiang Y, Li M (2019) Serious challenges and potential solutions for the industrial internet of things with edge intelligence. IEEE Netw 33(5):41-45

4. Liang F, Wei Yu, Liu X, Griffith D, Golmie N (2020) Toward edge-based deep learning in industrial Internet of Things. IEEE Internet Things J 7(5):4329-4341

5. Deng S, Xiang Z, Zhao P, Taheri J, Gao H, Yin J, Zomaya AY (2020) Dynamical resource allocation in edge for trustable Internet-of-Things systems: a reinforcement learning method. IEEE Trans Ind Inf 16(9):6103-6113

6. Piao Z, Peng M, Liu Y, Daneshmand M (2019) Recent advances of edge cache in radio access networks for Internet of Things: techniques performances, and challenges. IEEE Internet Things $\mathrm{J}$ 6(1):1010-1028

7. Yang J, Zhang J, Ma C, Wang H, Zhang J, Zheng G (2020) Deep learning-based edge caching for multi-cluster heterogeneous networks. Neural Comput Appl. https://doi.org/10.1007/s00521019-04040-z

8. Tang Q, Lyu H, Han G, Wang J, Wang K (2020) Partial offloading strategy for mobile edge computing considering mixed overhead of time and energy. Neural Comput Appl. https://doi. org/10.1007/s00521-019-04401-8

9. Song Y-j, Zhou Z-y, Zhang Z-s, Yao F, Chen Y-w (2020) A framework involving MEC: imaging satellites mission planning. Neural Comput Appl. https://doi.org/10.1007/s00521-019-040476

10. Li J-q, Zhang Y-f, Chen Z-z, Wang J, Fang M, Luo C-w, Wang H (2020) A novel edge-enabled SLAM solution using projected depth image information. Neural Comput Appl. https://doi.org/ 10.1007/s00521-019-04156-2

11. Sun L, Zhao Y, Sun W, Liu Z (2020) Study on supply chain strategy based on cost income model and multi-access edge computing under the background of the Internet of Things. Neural Comput Appl. https://doi.org/10.1007/s00521-019-041259

12. Ruan J, Xiangpei H, Huo X, Shi Y, Chan FTS, Wang X, Manogaran G, Mastorakis G, Mavromoustakis CX, Zhao X (2020) An IoT-based E-business model of intelligent vegetable greenhouses and its key operations management issues. Neural Comput Appl. https://doi.org/10.1007/s00521-019-04123$\mathrm{x}$

13. Liang H, Liu G, Gao J, Khan MJ (2020) Overflow remote warning using improved fuzzy c-means clustering in IoT monitoring system based on multi-access edge computing. Neural Comput Appl. https://doi.org/10.1007/s00521-019-04540-y

14. Zhou A, Wang S, Wan S, Qi L (2020) LMM: latency-aware micro-service mashup in mobile edge computing environment. Neural Comput Appl. https://doi.org/10.1007/s00521-019-04693w

Publisher's Note Springer Nature remains neutral with regard to jurisdictional claims in published maps and institutional affiliations. 